\title{
Modelling and Simulation of a Heat Exchanger1
}

\author{
Lei Xia, J. A. De Abreu-Garcia, and Tom T. Harley \\ Department of Electrical Engineering \\ The University of Akron \\ Akron, OH 44325-3904
}

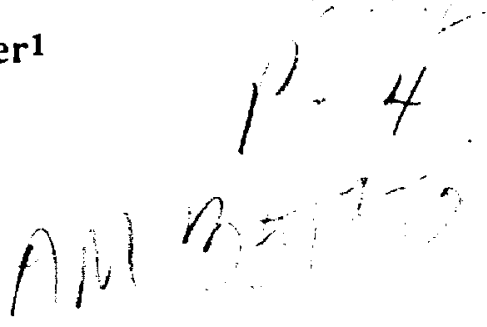

Abstract

Two models for two different control schemes are developed for a parallel flow heat exchanger. First by spatially lumping a heat exchanger model, a good approximate model which has a high system order is produced. Model reduction techniques are applied to these obtain low order models that are suitable for dynamic analysis and control design. The simulation method is discussed to ensure a valid simulation result.

\section{Introduction}

A heat exchanger is a device that is used to change the temperature distribution of two materials when they are in direct or indirect contact. They are used in many industries, particulerly in metallurgy, chemistry, etc [1]. A heat exchanger can be classified into two types based flow directions of two materials, namely, parallel flow and counter flow. Many heat exchangers being manufactured are basically open loop systems, i.e, the performance of the heat exchanger is determined by its fixed structural and mechanical design (e.g, size of compartment and tube, speed of cooling fan, etc.). If the performance (temperature distribution) of a heat exchanger deviates beyond the tolerance of the practical requirement, it has to be replaced by a new one.

Part of the reason for this situation is the lack of a suitable model for feedback control design. However, modelling a heat exchanger for dynamic analysis and control design is not an easy task for the following reasons.

(1) As will be seen, the dynamics of the heat exchanger is described by a partial differential equation, thus it is truly an infinite demesional system, which makes it difficult for the theories developed for lumped systems to be applied.

(2) Using some approximate techniques, the resulting system with acceptable accuracy usually has too high of order for easy dynamic analysis and control design.

This paper is a study of a parallel flow heat exchanger and solves the above problems with available techniques. The same techniques can be easily applied to counter flow heat exchangers.

\section{Modelling of a Heat Exchanger}

Parallel Flow Heat Exchanger

The following is an illustrative figure of a double-pipe parallel flow heat exachanger [1].

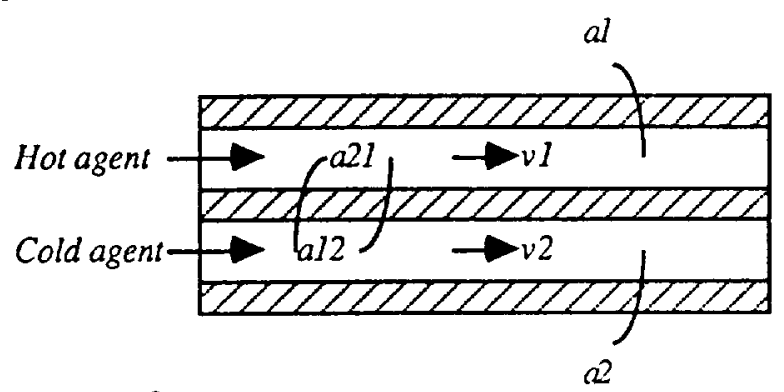

$Q$

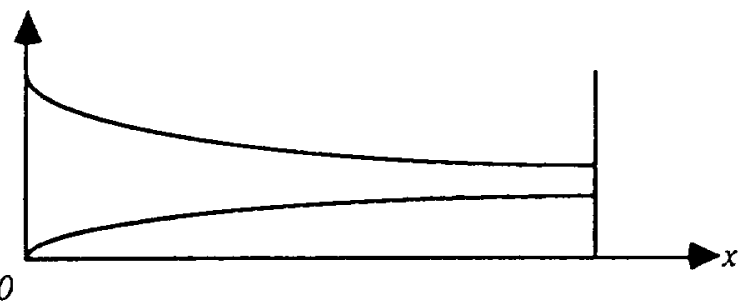

Ignoring the influence of of the partition wall (thin wall exchanger) the temperature distribution is given by :

$\frac{\partial Q_{1}(x, t)}{\partial t}=v_{l} \frac{\partial Q_{1}(x, t)}{\partial x}-a_{1} Q_{1}(x, t)+a_{2 l}\left(Q_{2}(x, t)-Q_{1}(x, t)\right)$
$\frac{\partial Q_{2}(x, t)}{\partial t}=v_{2} \frac{\partial Q_{2}(x, t)}{\partial x}-a_{2} Q_{2}(x, t)+a_{21}\left[Q_{1}(x, t)-Q_{2}(x, t)\right]$

where $v_{1}, v_{2}$ are the velocities of the two imcompressible flows, and $a_{1}, a_{2}, a_{2 l}$ are heat conductivity coefficients.

In what follow's, it is also assumed that the exchanger is well isolated, i.e, $a_{1}=a_{2}=0$. Upon these assumptions, the dynamic equations for the parallel flow heat exchanger are then:

$$
\begin{aligned}
& \frac{\partial Q_{1}}{\partial t}=v_{1} \frac{\partial Q_{1}}{\partial x}-a_{21} Q_{1}+a_{21} Q_{2} \\
& \frac{\partial Q_{2}}{\partial t}=v_{2} \frac{\partial Q_{2}}{\partial x}-a_{21} Q_{2}+a_{21} Q_{1}
\end{aligned}
$$

1. This research was partially supported by the Advanced Control Technology Branch of NASA Lewis Research Center via NASA Grant NAG 3-904. 
Control problem statement.

The control problem related to the heat exchanger here is to regulate the temperature of the cold medium at its outlet. In order to achieve this control goal, there are at least two control schemes that can be adopted. The first one is to use the temperature of the hot medium at its inlet as the control signal (input scheme 1), while the other is to use the flow rate of the hot medium as the control signal (input scheme 2). It should be pointed out that although almost all the existing models are derived for the first control method [2], this method suffers from the time delay to change the temperature of the hot medium. In contrast, the second control method is easier to implement in practice because the flow rate is manipulated by mechanical devices. Therefore obtaining a model for the second control method, which the authors have not seen in most of the related literature, has practical significance, again assuming imcompressible flow.

For either control method mentioned above, a system model (transfer function) needs to be developed for dynamic analysis and control design. As the system described by (2) is a Distributed Parameter System (DPS), it is then essentially an infinite demensional system, which makes it difficult for the control methods developed for finite lumped systems to be applied. In [3], a very complicated exact solution invloving many irrational functions is given for the input scheme 1. This model, however, is very difficult to use for practical control design. Many efforts [4,5] have been made to simplify this transfer function, but they are still infinite demensional. An attempt was made in [6] to approximate the heat exchanger purely by a polynomial function. However because there the authors used only two lumps to represent the whole exchanger, the results are predictably too rough for many practical uses. In fact, the inherent time delay property of the heat exchanger is completely lost in that model.

This paper proposes an another way for obtaining a system model which has adequate accuracy, low system order, and involves only polynomial functions.

\section{System Modelling}

Partition the length of the heat exchanger into small lumps uniformly at $h, 2 h, 3 h, \ldots, N h$, where $h$ equals the unit segment of $x$, i.e,

$$
h=\Delta x
$$

Define the system state to be the temperature at each lump as:

$$
x=\left[Q_{2}^{1} \ldots Q_{3}^{1} Q_{n}^{1} Q_{2}^{2} Q_{3}^{2} \ldots Q_{n}^{2}\right]
$$

where $Q_{j}^{p}(p=1,2, j=1,2, \ldots, N)$ is the $j^{\text {th }}$ node of the $p^{t h}$ tube. derivation:

Use the following approximation for spatial

$$
\frac{\partial Q_{j}}{\partial x}=\frac{Q_{j+1}-Q_{j}}{h}
$$

Then for input scheme 1, the normalized original system (2) $\left(v_{l}=v_{2}=a_{2 l}=1\right)$ is approximated by:

$$
\begin{aligned}
& \dot{Q}_{j}^{l}=\frac{Q_{j+l}^{l}-Q_{j}^{l}}{h}+Q_{j}^{2}-Q_{j}^{l} \\
& \dot{Q}_{j}^{2}=\frac{Q_{j+l}^{2}-Q_{j}^{2}}{h}+Q_{j}^{l}-Q_{j}^{2}
\end{aligned}
$$

It is easy to write the above equations in the form:

where:

$$
\dot{x}=A x+B u
$$

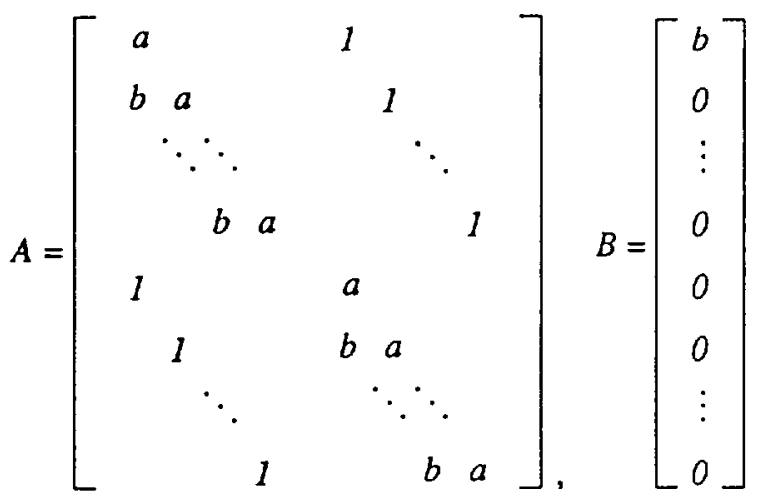

where $a=1 / h, b=-1-1 / h$, and $u=Q l$ is the system input.

Therefore the system order is $2 N-2$, where $N$ is the lump number of each tube. Figure 1 show the step response of the approximate open loop system with $40,20,10,4,2$ lumps, respectively, for $h=.025, .05, .1, .25, .5$ and $T=.0249, .0499, .0999, .2499, .4999$. It is seen that the response of the system with 40 lumps is very close to that of exact solution [3].

For input scheme 2, $v_{1}$ is used as the control signal. However $v_{l}$ is coupled with the system state $Q^{I}$ (sce (2)), using the same derivation as above will result in a time varying system. To avoid this problem, the steady states of $\partial Q_{j}^{1} / \partial x$ are used to replace the time varying $\partial Q_{j}^{\prime} / \partial x$, i.e, the system (2) is approximated by the following form:

$$
\begin{aligned}
& \dot{Q}_{j}^{l}=Q_{j}^{2}-Q_{j}^{l}+\left.\frac{\partial Q_{j}^{l}}{\partial x}\right|_{s s v_{l}} \\
& \dot{Q}_{j}^{2}=\frac{Q_{j+1}^{2}-Q_{j}^{2}}{h}+Q_{j}^{l}-Q_{j}^{2}
\end{aligned}
$$

This model is then valid for small perturbations from the given steady states.

The state space form of the system is: where:

$$
\dot{x}=A x+B u
$$




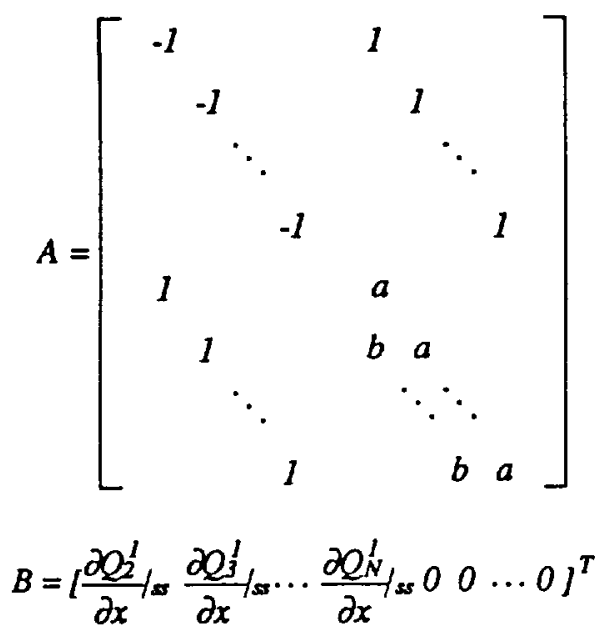

and $u=v_{1}$ is the system input.

In both schemes, the system output vector is:

$$
C=\left[\begin{array}{lllllllll}
0 & 0 & \ldots & 0 & 1 & 0 & 0 & \ldots & 1
\end{array}\right]
$$

Figure 2 shows the seady states of system, which are used in the input scheme 2. The step response of the approximate open loop system with the $N$ being $40,20,10$, 4,2 and the same $h$ and $T$ as above for input scheme 2 are shown in figure 3 .

\section{Model reduction}

The higher order model, though a good approximation of the original system, is not practical for control design due to its high (80th) order. Using model reduction techniques, these high order system can be reduced to lower order models while keeping the original system properties.

For the model corresponding to input scheme 1 , the real part of the eigenvalues of the system descend gradually from -53 to -30 , and do not display distinct time scales. But their Hankel singular values decrease fairly fast with the first seven of them being: $.3398, .1891, .0743, .0217, .0049,0,0, \ldots$

Thus keeping the first four states [7] results in a fourth order system with transfer function:

$$
G(s)=\frac{-.045 s^{3}+1.1875 s^{2}-13.5142 s+64.9335}{s^{4}+7.3217 s^{3}+47.3383 s^{2}+124.6222 s+159.7031}
$$

The step response of this reduced order system is shown in figure 4, where it is seen that it is a very good approximation to the previous 80 th order system. It should be emphasized that the reduced order system is much better than the system of the same order obtained directly by using less corresponding lumps as also shown in figure 4.

For the model corresponding to input scheme 2, the eigenvalues of the system are located in two groups which have distinctive time scales. One group of eigenvalues is located around $-40 \pm 10 j$, while the other group at $-.98 \pm .006 j$. Applying the sigular perturbation method [8] to the 80 th order model results in a second order system with transfer function:

$$
G(s)=\frac{.2 s^{2}-11.1876 s+34.0546}{s^{2}+1.8236 s+.8325}
$$

The step response of this system is shown in figure 5 and can be compared with the previous 80th order system and the same order system but obtained from less lumps. Again this reduced order model is a better approximation to the original system.

\section{System Simulation}

It is important for computer simulation to reflect the inherent system properties correclly. Realizing that the characteristic equation contains the most important inforamtion about the system, good simulation can only bc achieved by mapping the $s$-plane properties of the system into z-plane. The simulation described in the paper is guided by the techniques developed in [9]. These techniques have distinct merits in analyzing a simulation method for DPS in terms of its efficiency, intuition and convenience of use.

Due to the limited space, only the guidelines as to the choice of $T$ and $h$ are given as follows. Readers who are interested in the detailed analysis are recommended to consult further references in [9].

(1) $T \leq h$ is the neccessary condition for stability due to CFL condition:

(2) $T=h$ gives the best results;

(3) when $T=h$, the smaller $T$ is, the better the results

will be due to the damped second eigenvalue;

(4) In practice, $T$ is chosen slightly smaller than $h$ to avoid

oscillation due to the discretization of the system.

\section{Summary}

This paper proposes an alternative way to obtain two simple, fairly accurate models for the heat exchanger. It is concluded that using model reduction techniques applied to highly accurate finite difference approximations is much better than using low order (small number of lumps) finite difference approximations.

\section{References}

[1] Butkovskiy, A. G, "Structural Theory of Distributed system", Ellis Horwood Limited, 1983

[2] Welty, J. R., Engineering Heat Exchanger, Wiley. New York, 1978.

[3] Gorecki, H., Fuksa, S., Grabowski, P. and Korytowski, A., Analysis and Synthesis of Time Delay Systems, John Wiley \& Sons, 1978

[4] Paynter, H.M and Takahashi, Y. "A New Method of 
Evaluating Dynamic Response of Counter Flow and Parallel Flow Heat Exchanger", Trans. ASME, 78, 1956, pp.749

[5] Friedly, J. C., "Asymptotic Approximation to Plug Flow Process Dynamics", JACC, June, 1967

[6] Shoureshi, R. and Paynter, H. M., "Simple Models for Dynamics and Control of Heat Exchanger", Proceedings of the 27th CDC, Austin, Texas, Dec, 1988

[7] Moore, B. C., "Principal Component Analysis in Linear Systems: Controllability, Observability, and Model

Reduction", IEEE Trans. on Automatic Control, vol. ac26, 1981

[8] Kokotovic, P. V. and Khalil, H. K., "Singular Perturbation in Systems and Control", IEEE Press, New York, 1986

[9] Hartley, T.T., "A Qualitative Theory for Simulating Distributed Parameter Systems", Proc. of IECON'89, 1989

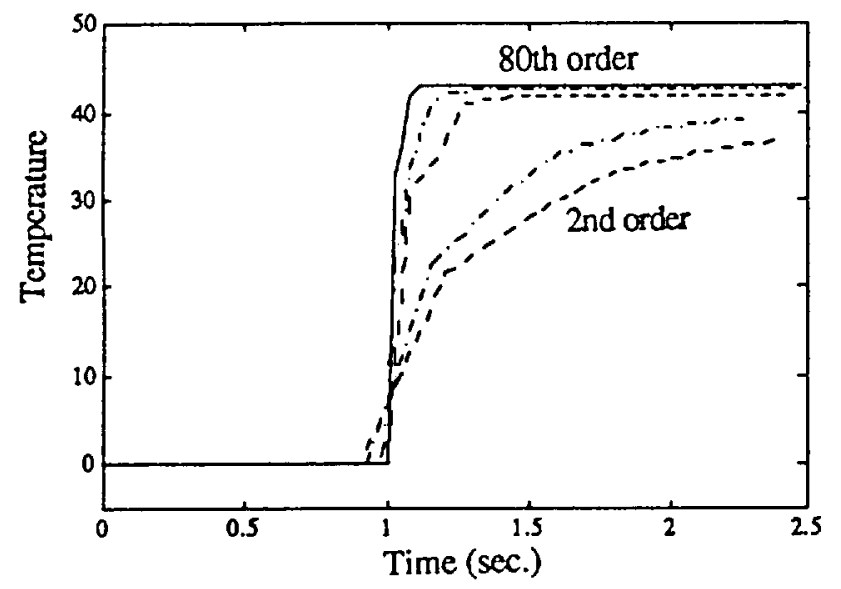

Fig. 1 Step responses of different order systems (schemel)

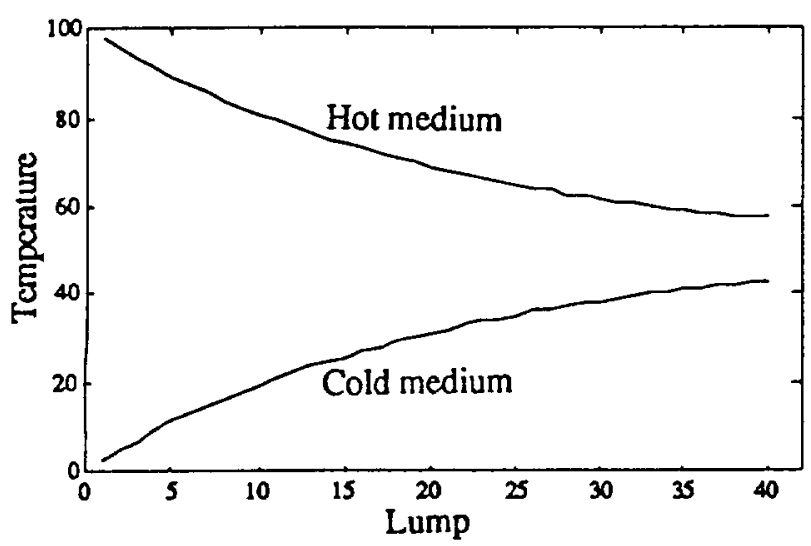

Figure 2 Steady states of the system response

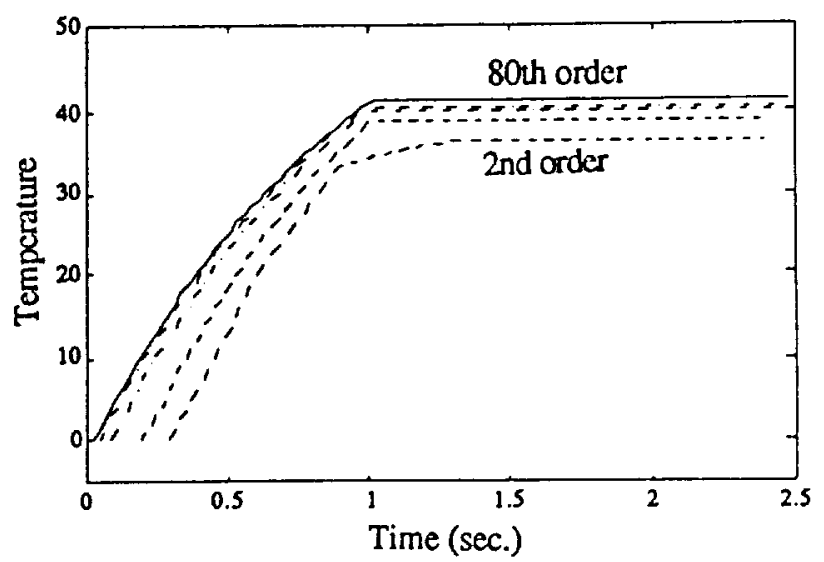

Fig. 3 Step responses of different order systems (scheme 2)

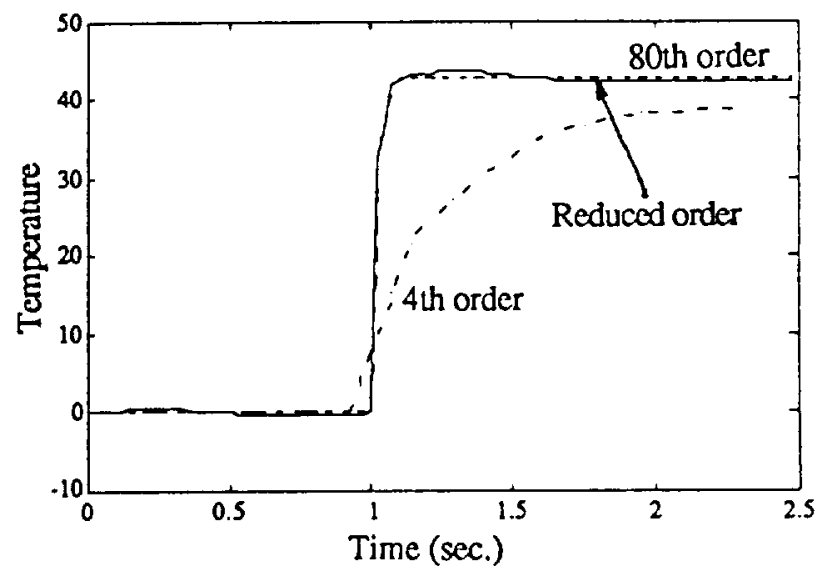

Figure 4 Comparison of different system models (scheme 1)

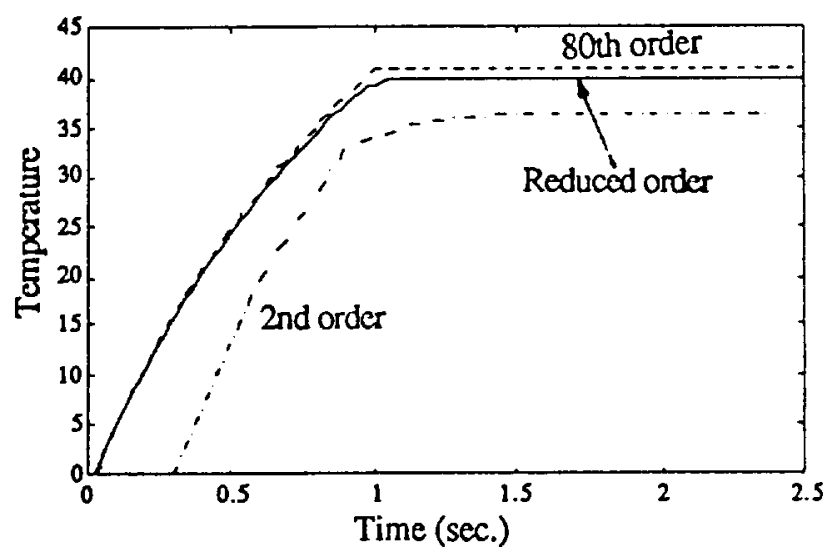

Figure 5 Comparison of different system models (scheme 2) 